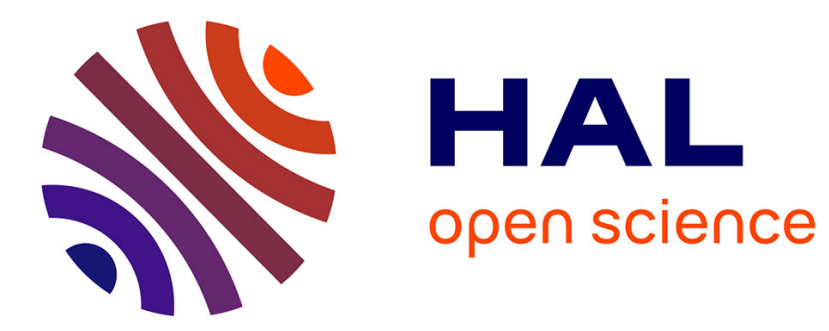

\title{
Spiking dynamics of interacting oscillatory neurons
}

\author{
V. B. Kasantsev, V. Nekorkin, S. Binczak, S. Jacquir, J. M. Bilbault
}

\section{To cite this version:}

V. B. Kasantsev, V. Nekorkin, S. Binczak, S. Jacquir, J. M. Bilbault. Spiking dynamics of interacting oscillatory neurons. Chaos -Woodbury-, 2005, 15 (2), pp.023103. 10.1063/1.1883866 . hal-00584216

\section{HAL Id: hal-00584216 \\ https://u-bourgogne.hal.science/hal-00584216}

Submitted on 8 Apr 2011

HAL is a multi-disciplinary open access archive for the deposit and dissemination of scientific research documents, whether they are published or not. The documents may come from teaching and research institutions in France or abroad, or from public or private research centers.
L'archive ouverte pluridisciplinaire HAL, est destinée au dépôt et à la diffusion de documents scientifiques de niveau recherche, publiés ou non, émanant des établissements d'enseignement et de recherche français ou étrangers, des laboratoires publics ou privés. 


\title{
Spiking dynamics of interacting electronic neurons
}

\author{
V.B. Kazantsev and V.I. Nekorkin \\ Institute of Applied Physics of RAS, \\ 46 Uljanov str., \\ 603950 Nizhny Novgorod, Russia
}

S. Binczak, S. Jacquir, and J.M. Bilbault

Laboratoire LE2I, Aile des Sciences de l'ingénieur, Université de Bourgogne, BP 47870 , 21078 Dijon Cedex, France

(Dated: June 23, 2004)

\begin{abstract}
Spiking sequences emerging from dynamical interaction in a pair of electronic neurons is investigated theoretically and experimentally. The model comprises two unidirectionally coupled FitzHugh-Nagumo units with modified excitability (MFHN). The The first (master) unit exhibits a periodic spike sequence with a certain frequency. The second (slave) unit is in its excitable mode and responds on the input signal with a complex (chaotic) spike trains. We analyze the dynamic mechanisms underlying different response behavior depending on interaction strength. The spiking phase maps describing the response dynamics are investigated. Complex phase locking and chaotic sequences are analyzed. We show how the response spike trains can be effectively controlled by the interaction parameter and discuss the possibility of neuronal information encoding.
\end{abstract}




\section{INTRODUCTION}

One of the key problems in the understanding of brain functions is how the neurons represent and bind sensory information converging to the brain from different sensory channels [1-3]. Neurons exhibit electrical activity and communicate using spike (electrical pulse) sequences so that all the information is represented (encoded) in the characteristics of the sequences. The neurons are subserved by the synaptic interactions (chemical and electrical) providing the activity modifications from one cell to another. The resulting space-time patterns of electrical activity is believed to be a neuronal tool to represent and process the information [3]. A number of recent neurophysiological studies of the activity in different brain areas have shown that neuronal information "code" deals with the control of spike timing. Take, for instance, spiking synchrony and reset properties in the olivo-cerebellar dynamics [3-5], $\delta$-oscillation phase response in the thalamo-cortical system [6], spike-timing in cerebellar stellate cells [7], $\theta$-oscillation phase precessing in hippocampus $[8,9]$. It appears that not only spiking rate but also spiking phase is important. Many recent works have been devoted to the study of "spike-communication-code" [10-13]. It has been found that integro-resonant properties of neurons as nonlinear dynamical systems underly the inter-neuron communications. Getting external stimuli the neuron may respond differently depending on both characteristics of the stimulus and internal dynamical state.

¿From neuron modeling point of view the basic concept of inter-neuron communication deals with the neuron response on incoming information messages. A number of recent works have contributed a lot on this issue [14-16]. Among others the synchronous and chaotic response have been found in the different models. By varying the input frequency one can achieve various spike frequency locking modes and chaotic areas. It has been shown that the response can be characterized by the phase maps describing possible inter-spike timings.

In this paper we report on spike dynamics in the neuronal system comprising two units with a unidirectional interaction. The unit is described by the FitzHugh-Nagumo model [17] with modified excitability (MFHN) [13]. For some particular parameters its dynamics is similar to a Morris-Lecar system $[18,19]$. In contrast with classical FitzHugh-Nagumo model typically used for cardiac cells modeling [15, 16] the MFHN dynamics involves a number of separatrix loop bifurcations what will be essential for the response dynamics. 
We assume that the input spike sequence is fixed with a certain frequency and come from the master MFHN unit in its oscillatory mode. The second (slave) unit is in the excitable mode and responds only when the external stimulation is strong enough. The unidirectional coupling can be seen as a simplest tool to model a synaptic interaction. It is activated when an input pulse comes and provides a dynamic delay of the response. We focus on the analysis of response sequences depending coupling parameter. We provide theoretical results on the dynamic (bifurcation) mechanisms of different response and experimental results obtained using MFHN electronic circuits [20].

The paper is organized as follows. In Sect. II we describe the model and its implementation with analog electronic circuit. In Sect. III we define 1D point map describing the dynamics of spiking sequences. Section IV is devoted to the map analysis and possible attractors of the map. We analyze basic bifurcation scenarios leading to complex (chaotic) spike dynamics. We also present the experimental study of the phase maps obtained with two MFHN electronic circuits in master-slave configuration. In Sect. V we develop the spike number analysis that could be a tool for "spike-communication-code". Section VI contains brief discussion of the results.

\section{MODEL}

The dynamics of two udidirectionally coupled MFHN electronic neurons is described by the following equations

$$
\begin{aligned}
& \dot{u_{m}}=f\left(u_{m}\right)-v_{m}, \\
& \dot{v_{m}}=\epsilon\left(g\left(u_{m}\right)-v_{m}-I_{m}\right), \\
& \dot{u_{s}}=f\left(u_{s}\right)-v_{s}+d u_{m}, \\
& \dot{v_{s}}=\epsilon\left(g\left(u_{s}\right)-v_{s}-I_{s}\right) .
\end{aligned}
$$

The $u_{m, s}$-variable describes the evolution of the neuron membrane potential, $v_{m, s}$ mimics the dynamics of ionic currents of the master and slave units, respectively. The function $f$ has a cubic shape, $f(u)=u-u^{3} / 3$, the function $g$ is taken piece-wise linear, $g(u)=\alpha u$, if $u<0$, and $g(u)=\beta u$, if $u \geq 0$. The parameters $\alpha$ and $\beta$ control the shape and the location of the $v$-nullcline, hence the excitation threshold. We fix the values $\alpha=0.5, \beta=2$. Parameter $\epsilon$ defines the relative time scales of $u$ and $v$ variable. Parameter $I_{m, s}$ controls the neuron depolarization level. The coefficient $d$ describes the interaction, which is excitatory 

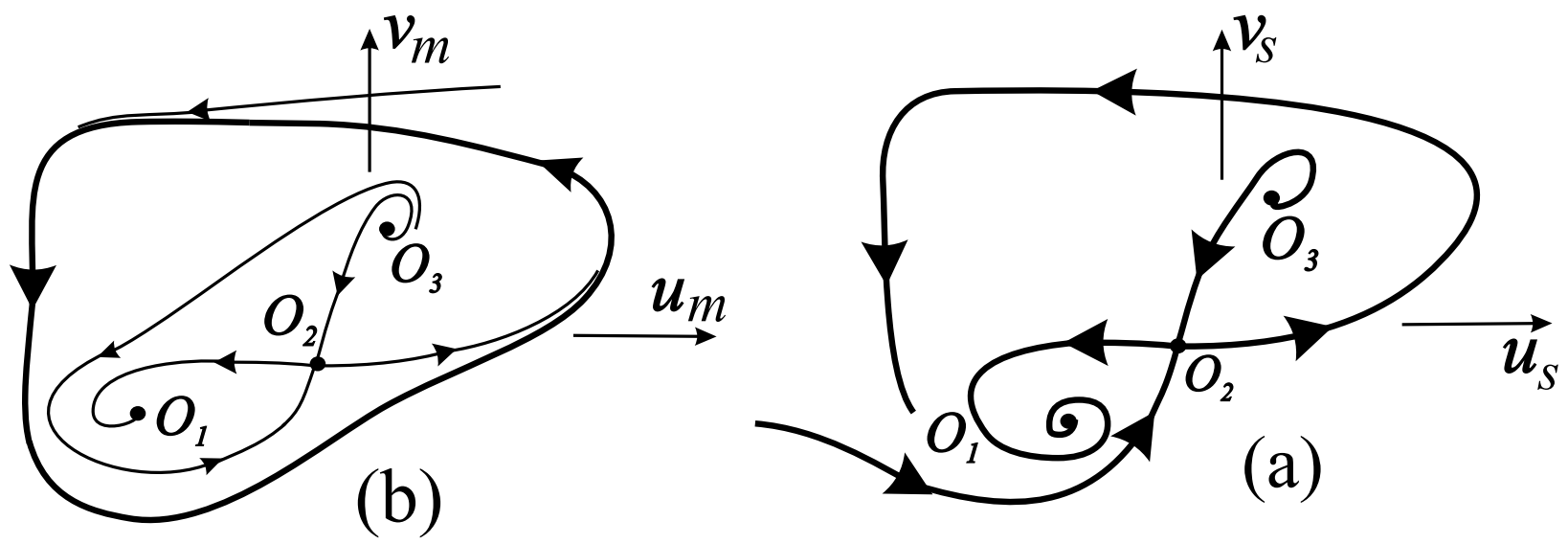

FIG. 1: (a) Phase portrait of the master unit dynamics. The unit has three fixed points and stable limit cycle corresponding to periodic spike train. (b) Excitable dynamics of the slave unit. The saddle separatrix defines the excitation threshold of the stable rest state $O_{1}$.

for $d>0$ and inhibitory for $d<0$.

The dynamics of MFHN units $(d=0)$ is illustrated on the phase plane shown in Fig. 1. The master unit (Fig. 1 (a)) has three fixed points and stable limit cycle for $I_{m}>I^{*}(\epsilon)$ corresponding to the periodic spike sequence. The slave unit is in its excitable mode, $I_{s}<$ $I^{*}(\epsilon)$, where $I^{*}(\epsilon)$ is a bifurcation parameter[? ]. It has stable fixed point, $O_{1}$, corresponding to the neuron rest potential, unstable fixed point, $\mathrm{O}_{3}$, and saddle point $\mathrm{O}_{2}$. The excitation threshold is defined by the saddle incoming separatrix. If the perturbation of the rest state is strong enough, i.e. lies below the separatrix curve, then the neuron responds with excitation pulse. The MFHN unit has been recently implemented with analog electronic circuit (FIG. 2 ). In this circuit, the voltage $U$ corresponds to variable $u$ by setting $u=R_{0} U$ where $R_{0}$ is equal to $R_{2} R_{4} / R_{3}$. The currents $I_{1}$ and $I_{2}$ are related to the variable $v$ in Eq. (1) by $v=\gamma R_{0}\left(I_{1}+I_{2}\right)$ where $\gamma$ is a fitting parameter so that the current voltage characteristic of part (A) is expressed by $I_{N L}=\frac{1}{R_{0}}\left[U-\frac{\gamma^{2} U^{3}}{3}\right]$. Thus, the dynamics of the circuit is given by Eq. (1) with $\epsilon=R_{0} R_{6} C / L_{1}, \alpha=R_{0} / R_{6}, \beta=R_{0}\left(L_{1}+L_{2}\right) /\left(R_{6} L_{2}\right), I=\gamma R_{0} E_{1} / R_{6}$ and time variable $t^{\prime}=t / R_{0} C$. The experimental phase portrait corresponding to the two theoretical cases (A) and (B) presented in Fig. 1 are shown in Fig. 3 and 4 respectively. 


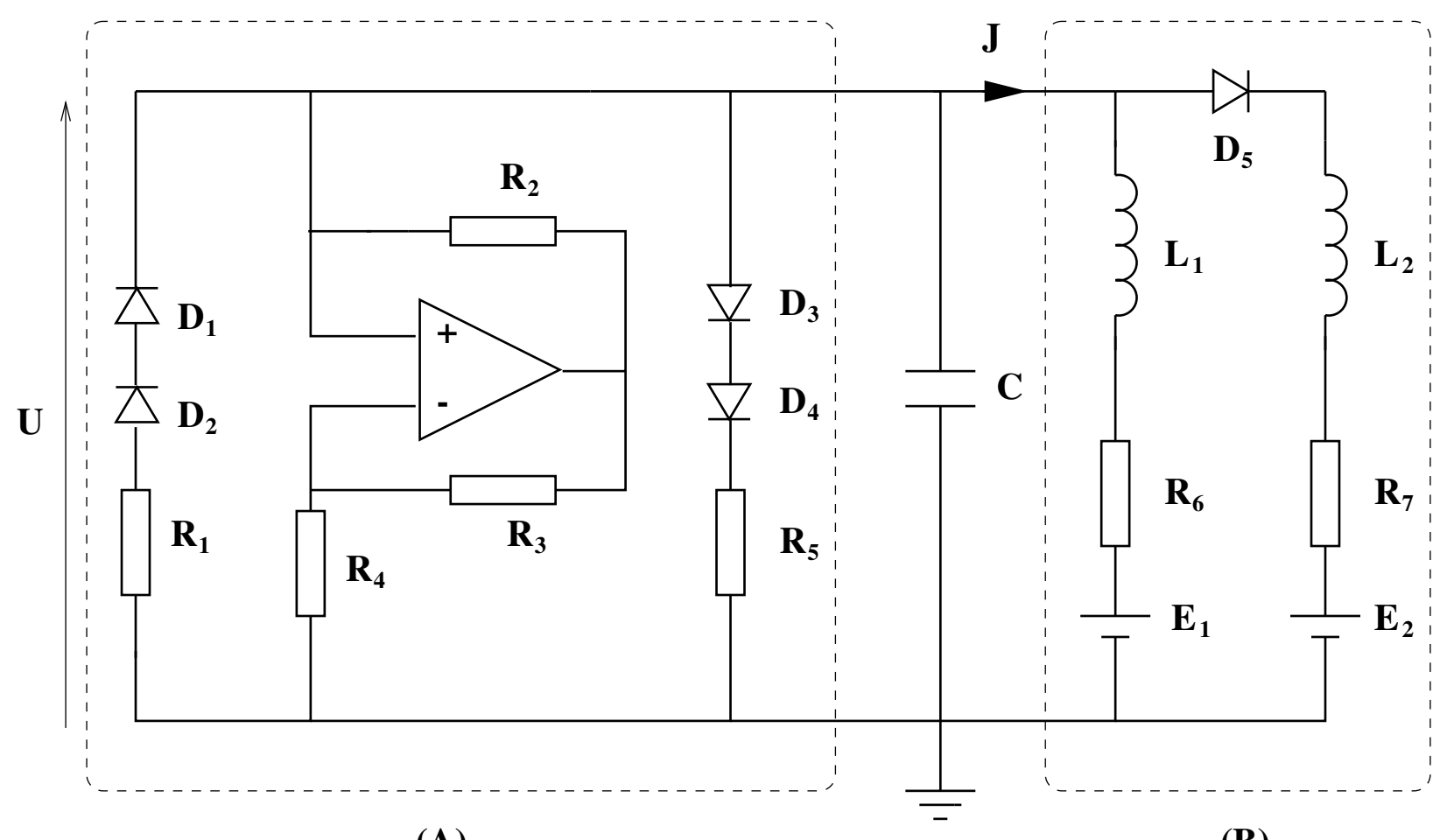

(A)

(B)

FIG. 2: MFHN electronic circuits. Electrical parameters : $R_{1}=500 \Omega, R_{2}=2 k \Omega, R_{3}=8 k \Omega$, $R_{4}=4 \mathrm{k} \Omega, R_{5}=1.2 \mathrm{k} \Omega$, leading to $R_{0}=1010 \mathrm{k} \Omega$ and $\gamma=1.138 \mathrm{~V}^{-1} . \quad L_{1}=10.2 \mathrm{mH}$, $L_{2}=3.5 \mathrm{mH}, R_{6}=2021 \Omega, R_{7}=690 \Omega$ and $E_{2}=-0.6 \mathrm{~V}$, leading to $\alpha=0.5, \beta=1.96$. For the oscillating (master) unit we set: $I=0.22\left(E_{1}=0.3876 \mathrm{~V}\right), \epsilon=0.2(C=1 n F)$. Parameters, for the excitable (slave) unit: $I=0.19\left(E_{1}=0.332 \mathrm{~V}\right), \epsilon=0.2(C=1 n F)$.

\section{DESCRIPTION OF RESPONSE DYNAMICS}

\section{A. Spiking phase sequences}

Let us consider the case of the excitatory interaction when the excitable unit gets a periodic spike train with $d>0$. Then, the dynamics of the slave unit is defined in the threedimensional phase space $\left(u_{s}, v_{s}, t\right)$ and can be very complex. Since the unit has excitation threshold for sufficiently small values of $d$ no response appear. In this case it displays lowamplitude subthreshold oscillations of periodic or chaotic shape. Let us consider $d>d_{c r}$ when spike response appears. To characterize the dynamics we introduce spiking phase map. As the response spikes appear due to periodic stimulation we can treat the master signal as a reference (base) signal. Let a master spike appears at the time moment $t_{n}^{m}$, and the 


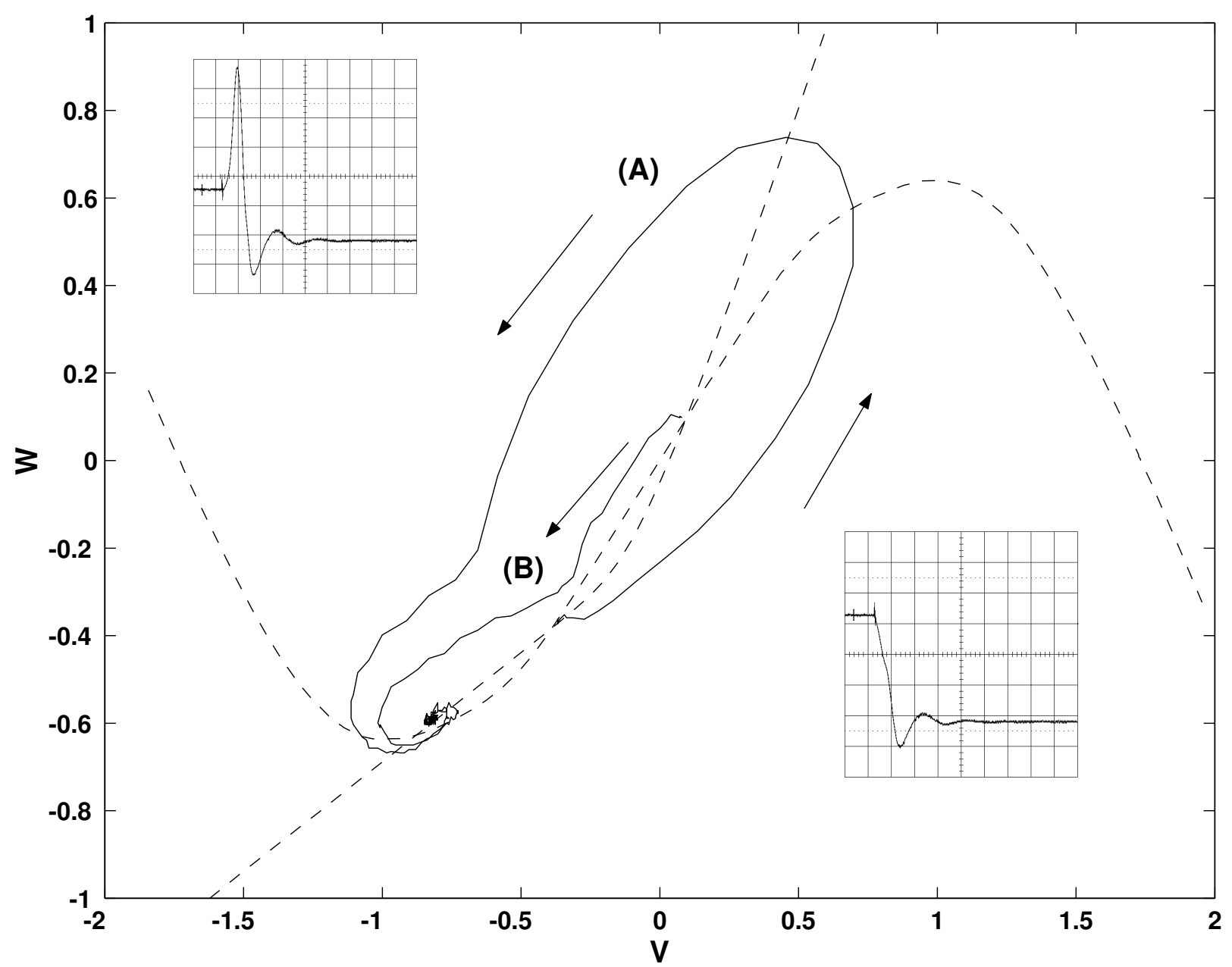

FIG. 3: Dimensionless phase plane (V,W) showing stability with excitation threshold. Parameters $: \eta=0.19$ and $\varepsilon=0.2\left(E_{1}=0.332 \mathrm{~V}\right.$ and $\left.C=1 n F\right)$. Insets: Excitation pulse (upper inset) corresponding to (a); perturbation reaching resting state (lower inset) corresponding to (b). Parameters of insets: abscissa $20 \mu \mathrm{s} /$ div; ordinate $225 \mathrm{mV} /$ div.

following response spike at the time moment $t_{n}^{s}$. Then, we define relative response spiking phase as

$$
\phi_{n}=\left(t_{n}^{s}-t_{n}^{m}\right) / T, n \in Z
$$

where $T$ is the period of the reference signal (Fig. 3(a)). If the response pulse appears at each reference period, then $0<\phi_{n} \leq 1$. If the slave unit responds "integrating" a certain number of the master's spikes, then $z_{n}<\phi_{n}<z_{n+1}$, where $z_{n}=\left[\phi_{n}\right] \in Z$ is the integer part of the spiking phase. FIGURE 3 (c) shows the spiking phase sequence plotted in $\left(\phi_{n}, \phi_{n+1}\right)$ - plane corresponding to the response shown in FIG. 3(b). The points are located in the 


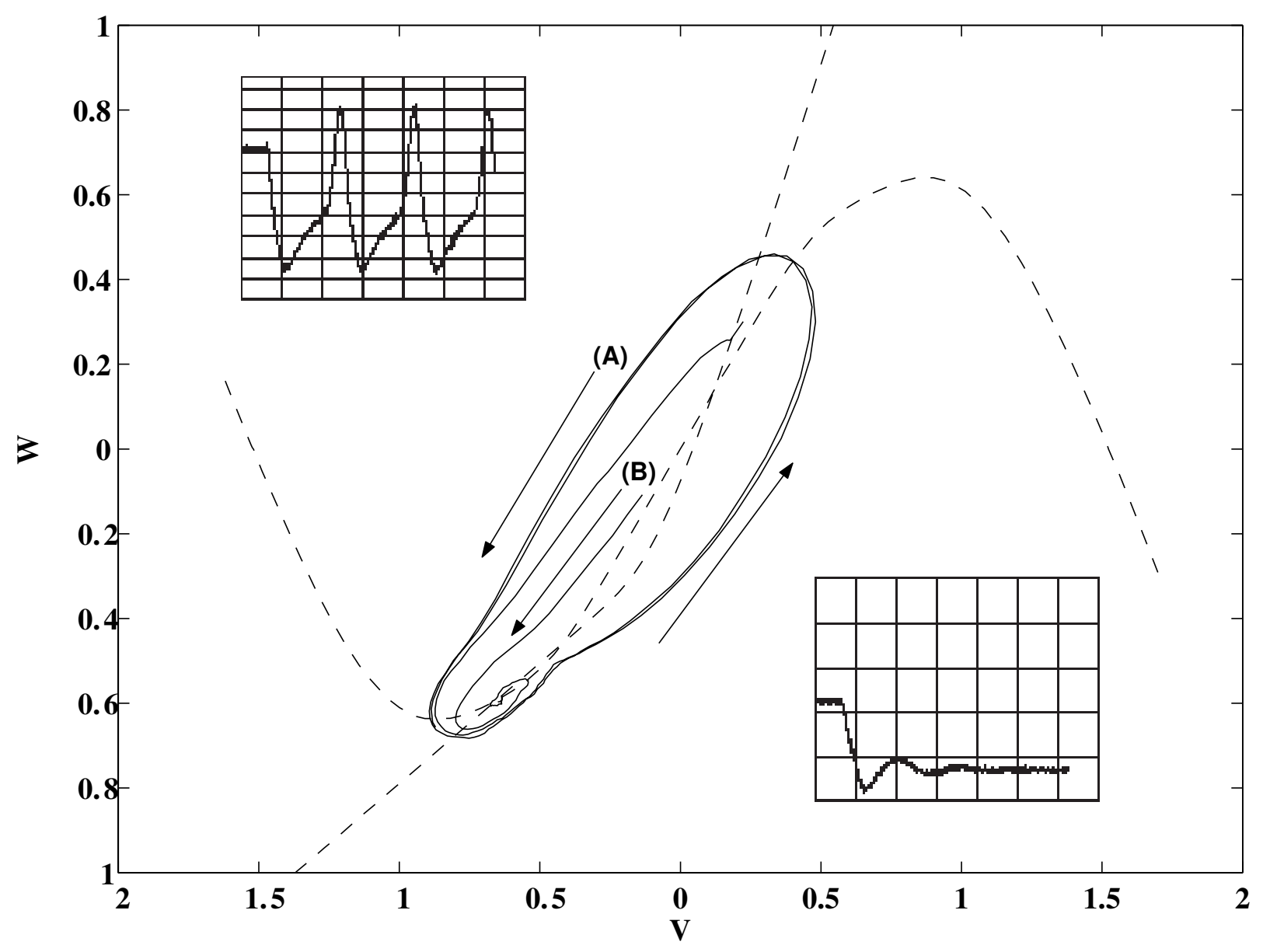

FIG. 4: Dimensionless phase plane (V,W) showing bistability. Parameters : $\eta=0.226$ and $\varepsilon=0.2$ $\left(E_{1}=0.3981 V\right.$ and $\left.C=1 n F\right)$. Inset: Spiking train of pulses (upper inset)corresponding to (A); perturbation reaching resting state (lower inset)corresponding to (B). Parameters of inset: abscissa $20 \mu s / d i v$; ordinate $500 \mathrm{mV} /$ div.

small neighborgood of some piece-wise continuous curve $\Pi\left(\phi_{n}\right)$ that is called phase response curve (PRC).

\section{B. Phase response curve}

The dynamics of the slave unit (1) is described by two-dimensional nonautonomous system with periodic forcing in the three-dimensional phase space $\left(u_{s}, v_{s}, t\right)$. The projection of its trajectories to the plane $\left(u_{s}, v_{s}\right)$ for the time series shown in FIG. 3 (b) is illustrated in FIG. 4 by dots. Note, that the points occupies a thin layer that attracts all trajectories 


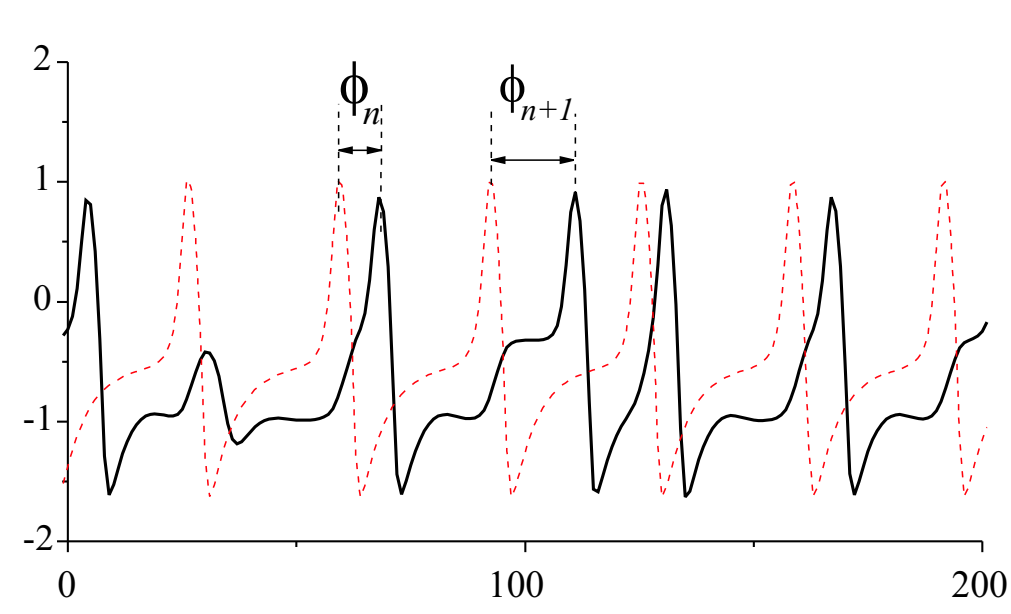

(a)

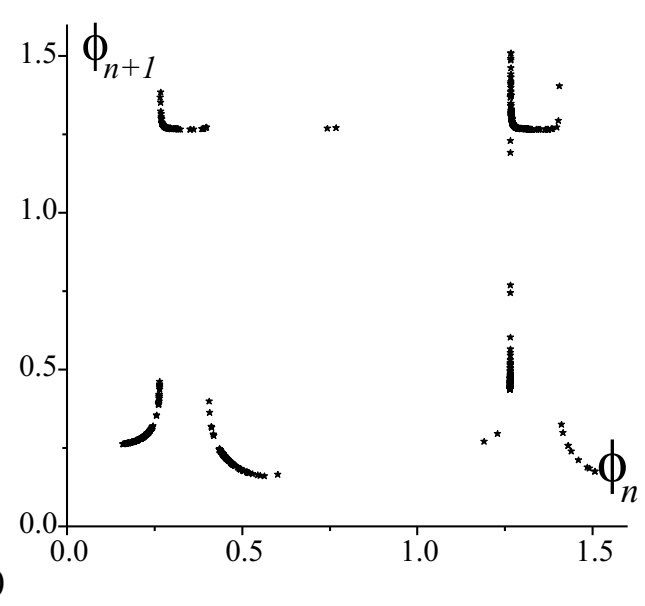

(b)

FIG. 5: (a) Fragment of superimposed master and slave time series shown by dashed and solid curves, respectively. The spiking phase, $\phi_{n}$, is defined as a time shift between master and slave oscillations peaks. Parameter values: $\epsilon=0.441, I_{m}=0.218, I_{s}=0.21$. (b) Spiking phase diagram showing the phases located in the neighborhood of some piece-wise continuous curve.

from neighborhood. The shape of the layer is similar to the shape of the limit cycle in the master unit (FIG. 4, dashed curve). Note also, that if response spike appear the points tend to curve interval, $L$, near the peak of the $u_{s}$-variable. To describe the spike dynamics let us fix a half-line, $M$, in the phase plane $(u, v)$ originating in some point $O$ located inside the master limit cycle and hitting the limit cycle near the peak point of $u$-variable (FIG. 4 (b)). Let us set the initial conditions, $t=0$, for the slave unit at the point $K_{s}$ corresponding to the intersection of $L$ and $M$. Then, let us set the master unit at the point of the limit cycle having the angle $\varphi$ with the half-line $M$ accounted counterclockwise and defined in the interval $[0,2 \pi]$. This angle is proportional to the time interval, $\tau_{0}$, between the master and slave spikes. As time proceed, the trajectories of system (1) map the $\left(u_{s}, v_{s}\right)$ variables back to the half-line $M$ in the neighborhood of the curve interval $L$. Let us denote this time interval by $\tau_{1}(\varphi)$. Then, at $t=\tau_{1}$ the master unit reaches the angle $\psi(\varphi)$. Therefore, the system trajectories define a point map $F: \varphi \rightarrow \psi$ defined in the interval $[0,2 \pi]$. As the angles are proportional to the spiking times the dynamics can be also expressed in terms of master-slave time differences $\Pi: \tau_{0} \rightarrow\left(\tau_{1}+\tau_{0}-T\right)$. By normalizing times on master oscillation period, $\phi_{n}=\tau_{0} / T, \phi_{n+1}=\left(\tau_{1}+\tau_{0}-T\right) / T$ we find the spiking phase map in the 
form

$$
\phi_{n+1}=\Pi\left(\phi_{n}\right)
$$

defined in the half-line $[0,+\infty)$ by the PRC function $\Pi\left(\phi_{n}\right)$. The map (3) describes two main characteristics of the spiking sequences. (i) The phase shift between the response spike and the closest preceding master spike. In this case the spiking phase, $\phi_{n}$, is taken on modulus 1. (ii) The number $z_{n}=\left[\phi_{n}\right]$ of integrated master spike to have the response. Note that the approximate 1D map description of the response dynamics becomes possible due to the existence of an curve interval $L$ approximating invariant curve on the $\left(u_{s}, v_{s}\right)$ section of non-autonomous 2D system. Note also that even if the return intersection point, $K_{s}$, appears with a shift relative to $L$ it further evolves with approximately the same phase. Such phase definition is similar to conception of isochrons recently introduced in [16].

\section{PHASE MAP ANALYSIS}

\section{A. Phase map attractors}

Let us analyze basic spiking modes of the response and bifurcation scenarios of their appearance. It has been found that for large values of $d$ the spike sequences from the master and slave units become synchronized with $1: 1$ spike frequency ratio. In this case slave spikes are phase-shifted relative to the master. The shift value vanishes with $d \rightarrow \infty$. This corresponds to the stable fixed point $0<\phi^{*}<1$ of the map (3). For smaller $d$ complex spikes modes appear. The bifurcation diagram illustrating the map attractors is shown in FIG. 5 (a). The two enlarged regions in FIGs. 5(b,c) show a fine structure of the diagram for particular intervals of $d$. It appears that the map attractors can have complex (chaotic) structures alternating with periodic windows of complex spike frequency locking ratios. Let us consider how the PRC evolves with changing $d$. We illustrate the dynamics using normalized phase variable, $\phi_{n}, \bmod 1$, defining the phase shift between the response spike and preceding master spike.

- For 1: 1 locking mode near the boundary of chaotic behavior the map (3) has stable and unstable fixed points in the neighborhood of saddle-node bifurcation (FIG. 6 (a)). 


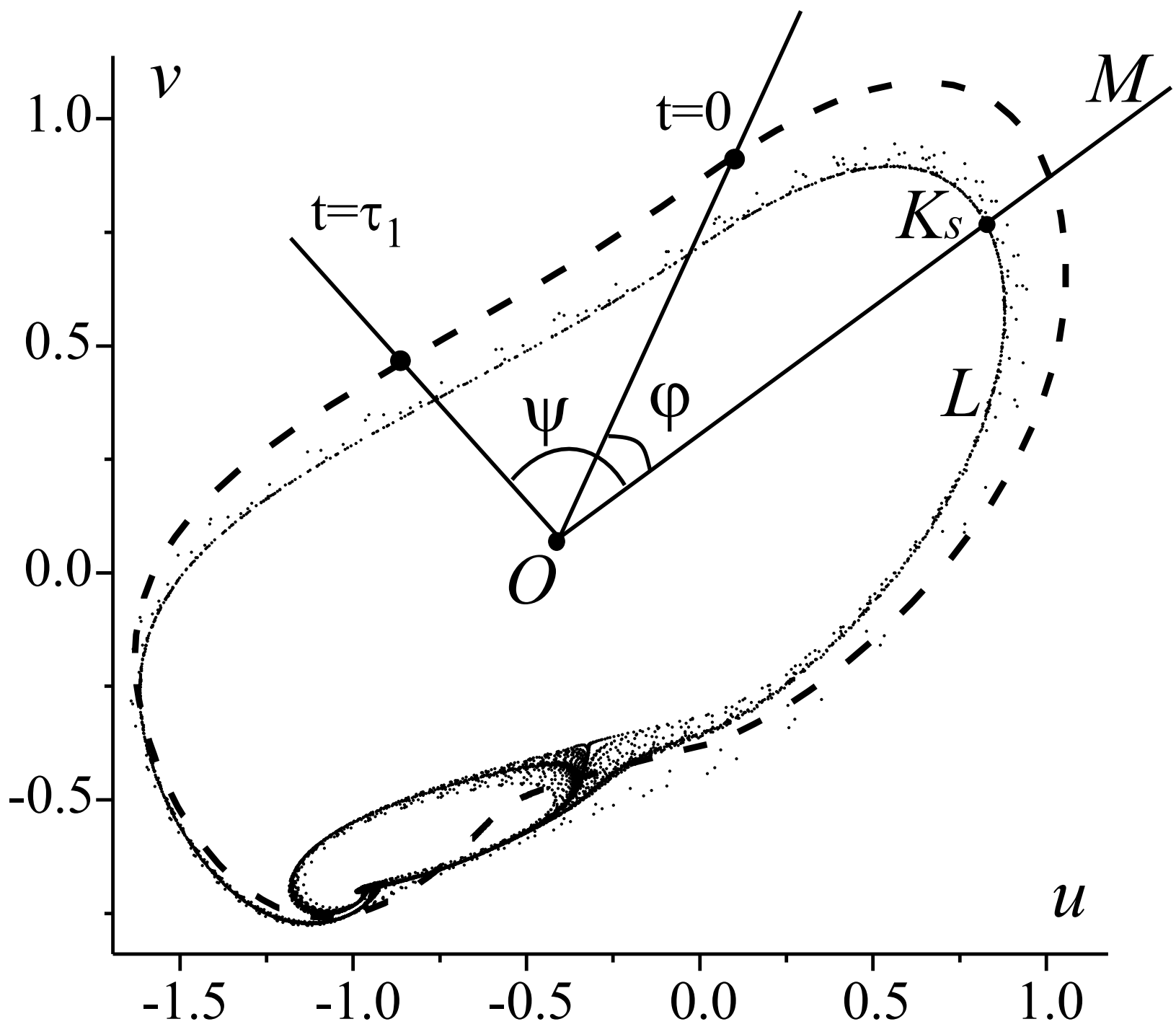

FIG. 6: Phase definition in the $(u, v)$-plane. The master limit cycle is shown by dashed curve. The dots show the $(u, v)$-projection of nonautonomous slave dynamics. They occupy a thin layer that we approximate by invariant curve interval $L$ (see text for details).

- Then, with decreasing $d$ the saddle-node bifurcation takes place and the two fixed points disappear (FIG. 6 (b)). However, the map curve stays very close to the bisector line where trajectories can stay for a long time. This corresponds to a long lasting 1 : 1 spiking when the inter-spike phase shift is monotonically increasing. Then, trajectories jump onto another part of the map curve corresponding to $2: 1$ mode. In one iteration this part is mapped back to saddle-node bifurcation region. It means 


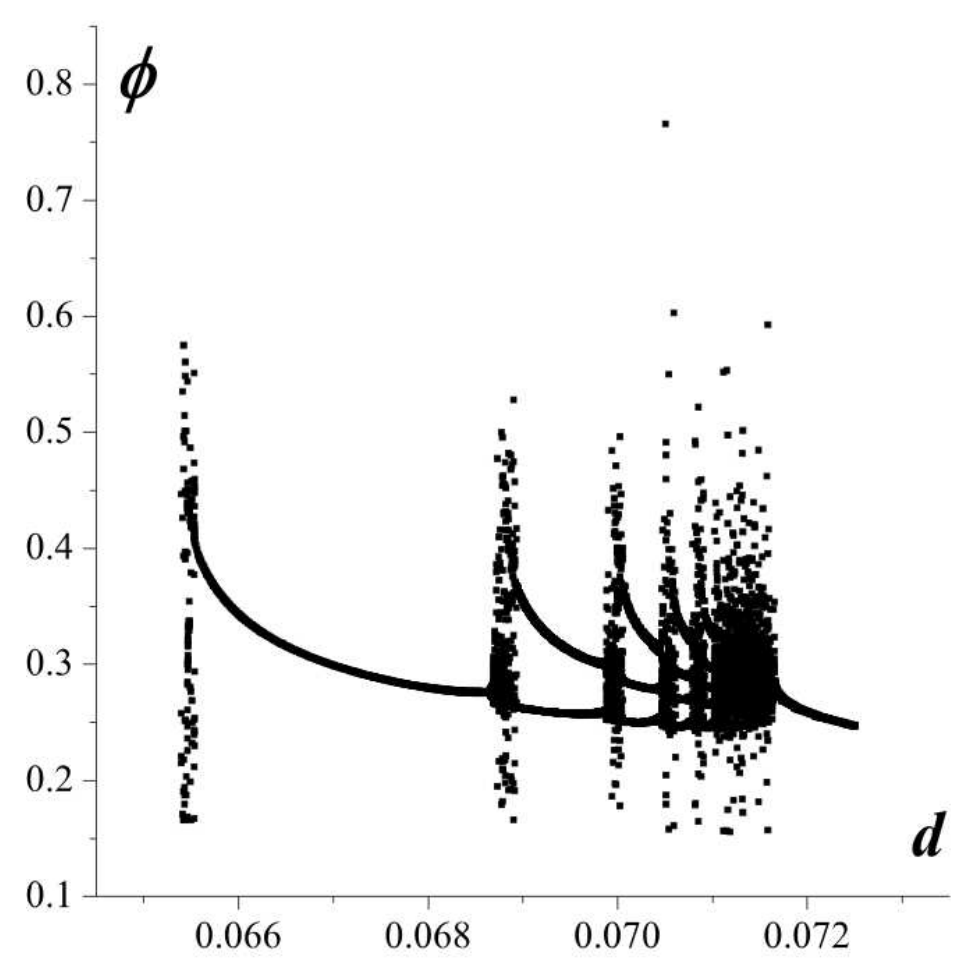

(a)

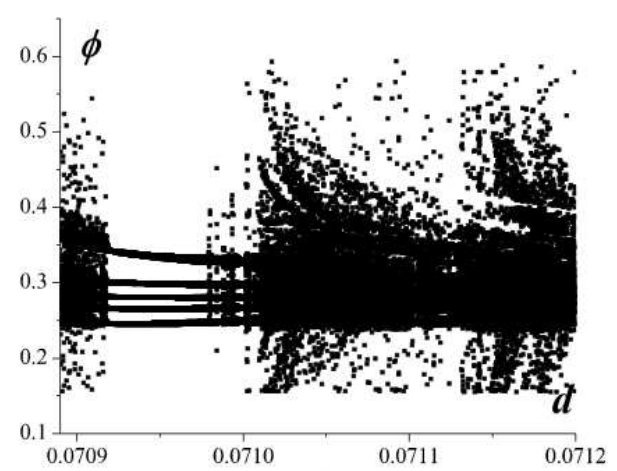

(b)

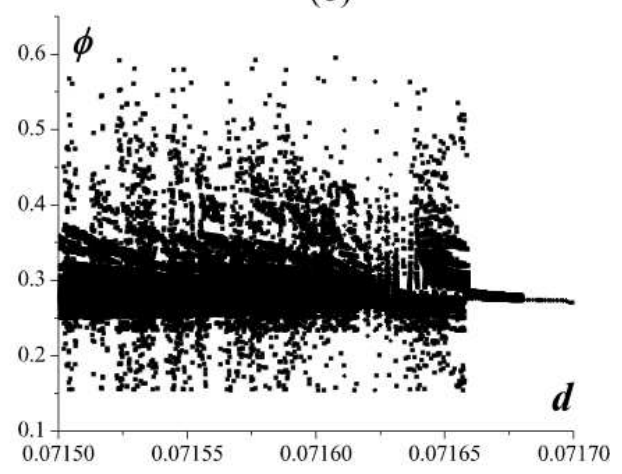

(c)

FIG. 7: (a) Spiking phase bifurcation diagram for normalized phase, $\phi_{n}, \bmod 1$, depending on interaction strength $d$. For fixed $d$ the dots show map attractors. (b), (c) Two enlarged regions of the diagram. Parameter values: $\epsilon=0.441, I_{m}=0.218, I_{s}=0.21$.

that after missing a one spike the response dynamics returns back to $1: 1$ region with increasing phase shift. Such map behavior corresponds to intermittency mechanism of chaotic dynamics. The regular ("laminar") phase is intermitted by jumps ("turbulent phase") that breaks the "laminar" dynamics. Chaotic response signal corresponding to map attractor (FIG. 6 (b)) is illustrated in FIG. 7 (a).

- Along with chaotic trajectories the map can have periodic windows with complex spike frequency locking. FIGURE 6 (c) illustrates the example of $6: 5$ mode when the slave unit responds with 5 spikes for each 6 master spikes.

- With further decrease of $d$ the number of map discontinuities increases. This corresponds to growing number of spikes to be missed in the response sequence (FIG. 7 (b)). In FIG. 6 (d) the map has one unstable fixed point, $P$, with the multiplier, $\mu(P)<-1$. This point belongs to the map compartment corresponding to $2: 1$ spike 
locking. When the multiplier approaches to the bifurcation value $\mu(P)=-1$ the map displays another type of intermittency (FIG. 6 (d)). Trajectories stay for along time rotating around the fixed point with increasing amplitude, then leave the region jumping over the discontinuity to another map compartment with different locking ratio. With changing $d$ the points $P$ acquires stability, $|\mu(P)|<1$ through the inverse period doubling bifurcation (FIG. 6 (e)).

- The left part of the bifurcation diagram on FIG. 5 (a) corresponds to a rare spiking response (FIG. 7 (c)). In this case has a large number of discontinuities corresponding to transitions between $M: 1$ spiking modes with $M=1,2,3, \ldots$. Map trajectories jumps from one mode to another forming a complex (chaotic) routes. Note that the continuous map compartment in the center corresponds to $1: 1$ ratio. The trajectory shown by solid circles hits this region at almost each cycle. Then, one can expect spike doublets in the response sequence what is clearly seen in FIG. 7(c).

Note that the present study does not cover all possible behaviors. In particular, we do not consider here various multi-stable spiking modes when two or more attractors co-exist in the map (3). Spiking modes may also co-exist with stable subthreshold responses. In this case the map (3) will have undefined intervals where no spiking response appears.

\section{B. Spiking phase map in electronic experiments}

The electronic circuit coupling the two electronic neurons is presented in Fig. 10.

\section{SPIKE NUMBER ANALYSIS AND SPIKE ENCODING}

We have studied the phase map attractors using normalized phase shift variable, $\phi_{n}, \bmod 1$. Let us consider another characteristics of the response sequence describing spike number dynamics, $z_{n}(d)=\left[\phi_{n}\right] \in Z$. This variable takes discrete values showing the number of slave spikes missed in the response sequence. In other words, it describes the number of "integrated" master spikes to get a response at the particular moment of time. So that the spiking sequences can be associated with sequences of digits. For example, simple frequency locking mode, $1: 1$, is associated with trivial sequence of zeros, $2: 1$ with periodic sequence 


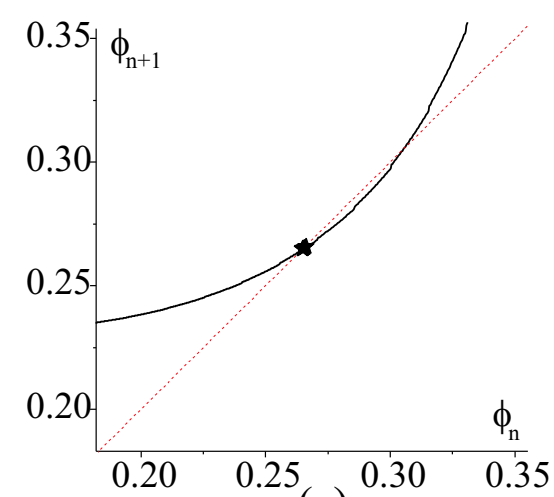

(a)

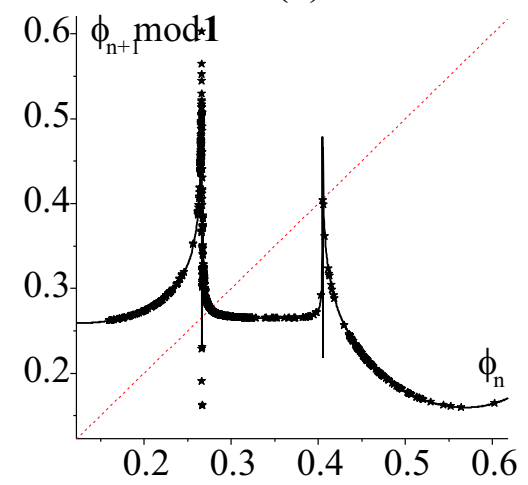

(d)

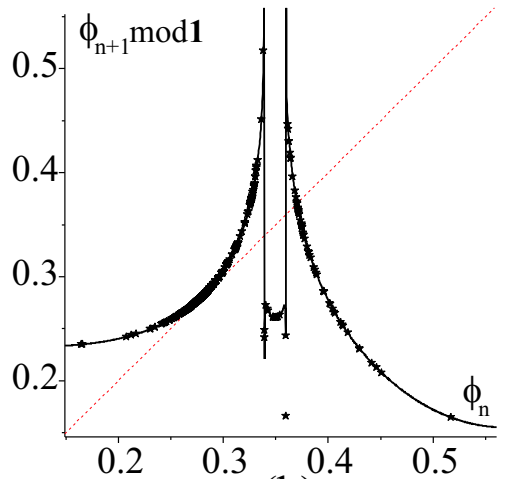

(b)

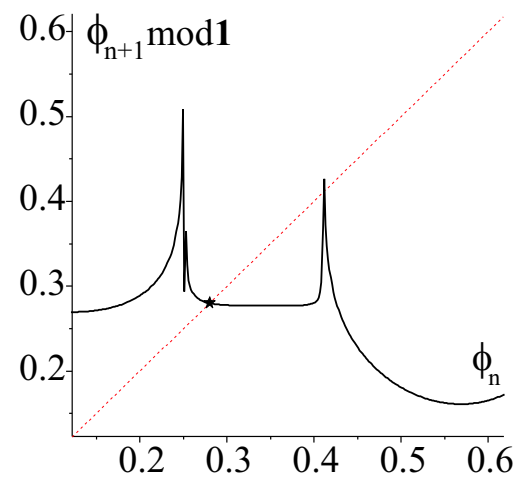

(e)

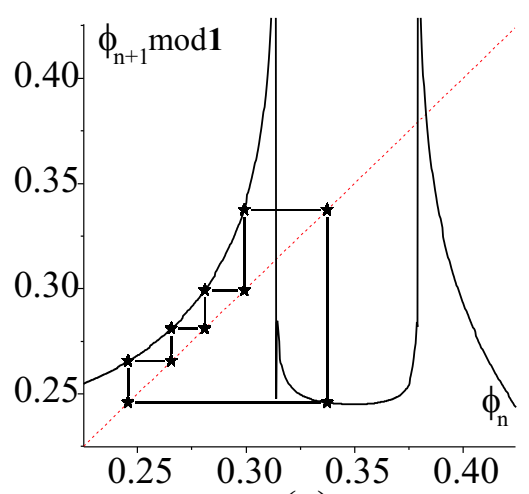

(c)

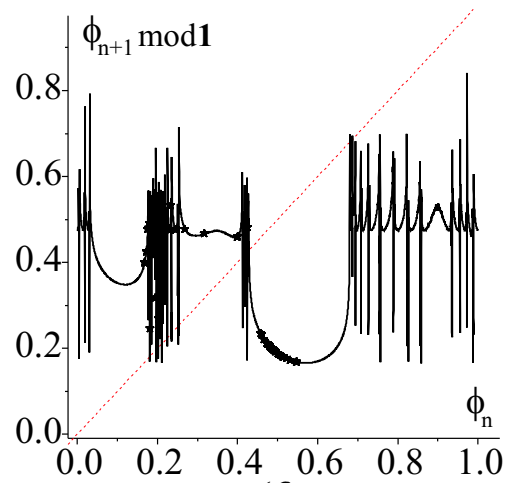

(f)

FIG. 8: PRC and corresponding map attractors shown by dots for different values of $d$. Parameter values: $\epsilon=0.441, I_{m}=0.218, I_{s}=0.21$. (a) Stable fixed point near saddle-node bifurcation, $d=0.07183$. (b) Intermittency attractor emerged from the saddle-node bifurcation, $d=0.0716$. (c) Stable periodic window with $6: 5$ spike locking ratio, $d=0.07093$. (d) Intermittency attractor near period doubling bifurcation, $d=0.06878$. (e) Stable fixed point corresponding to $2: 1$ spike locking mode, $d=0.068$. (f) Map attractor for large number of PRC discontinuities, $d=0.06543$. of " 1 " and so on.

$$
\begin{aligned}
& 1: 1 \rightarrow\{\ldots, 0,0,0,0,0,0,0,0,0,0, \ldots\} \\
& 2: 1 \rightarrow\{\ldots, 1,1,1,1,1,1,1,1,1,1, \ldots\} \\
& 3: 2 \rightarrow\{\ldots, 0,1,0,1,0,1,0,1,0,1, \ldots\} \\
& 6: 5 \rightarrow\{\ldots, 0,0,0,0,1,0,0,0,0,1, \ldots\}
\end{aligned}
$$

If the map (3) has one discontinuity (FIGs. 6 (a)-(c)) its dynamics can be described by binary sequences (4). Chaotic attractors (FIG. 6 (b)) of the map are described by chaotic sequences. The attractors, hence the sequences, are controlled by the value of $d$ (FIG. 5). In other words the sequences can be treated as a kind of "spike-communication-code" that 


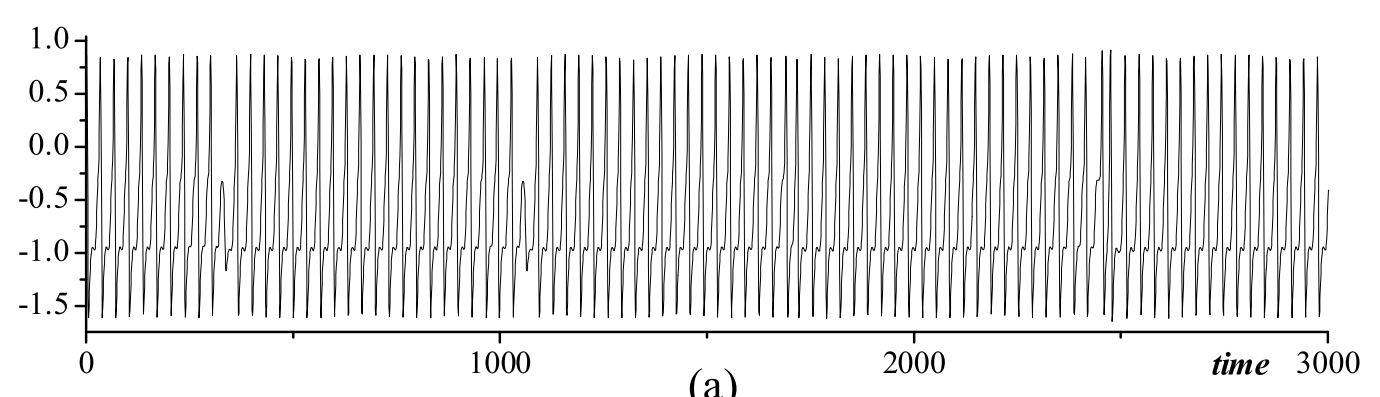

(a)

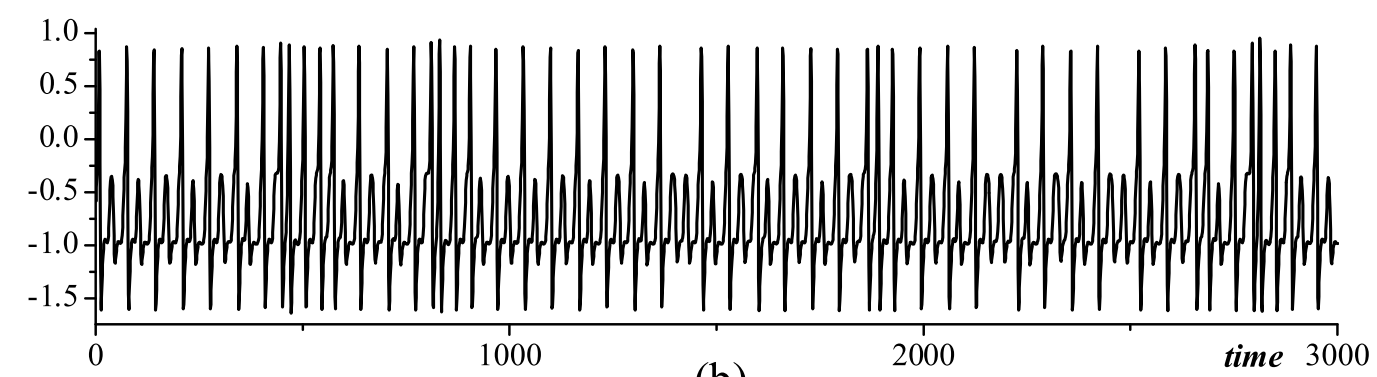

(b)

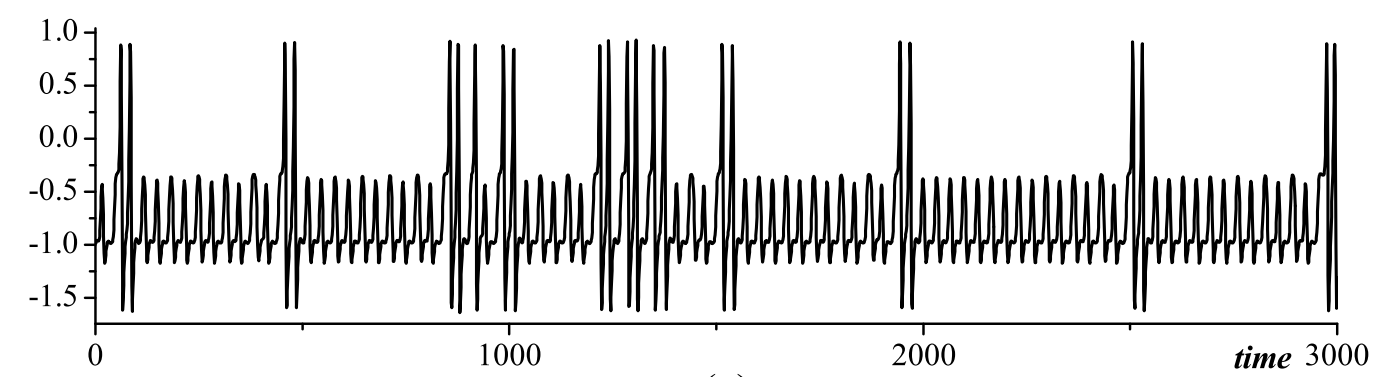

(c)

FIG. 9: Response time series for different values of $d$ (a) $d=0.0716$. (b) $d=0.06878$. (c) $d=0.06543$.

is driven by synaptic interaction, $d$. Note, that such a "code" can be "enhanced" up to an arbitrary number of digital levels. FIGURE (11) shows the bifurcation diagram for $z=[\phi]$. For lower values of $d$ the map trajectory passes a large number of the map compartments corresponding to different number of $z_{n}$ (FIGs. 6(f), 7(c)). The particular route is defined by the shape of the PRC which is given by piece-wise continuous function. The example of the route for FIG. 6 (f) is illustrated in FIG. (12). Then, such routes could be thought as neuronal "memorizing" tools using spiking "coding" space. A particular route type corresponds to definite value of interaction coefficient, $d$. In the case of chaotic attractors 


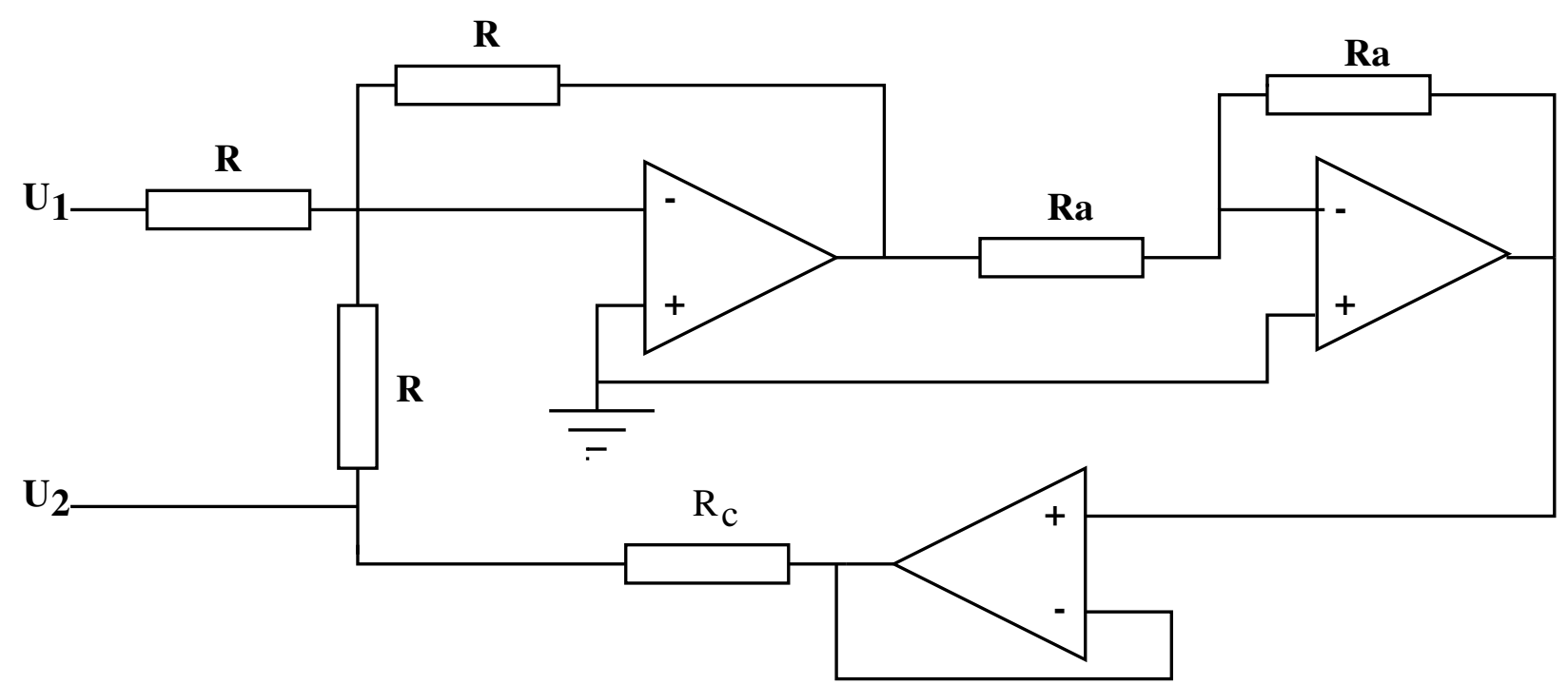

FIG. 10: Unidirectional coupling circuit

close trajectories diverge as time proceed, and the system "forgets" its initial state. However, for shorter time lengths (much less than the instability time scale) the digital route is welldefined and predictable. Thus in the chaotic case one can reset the initial condition, $z_{0}$, and deal with finite interval routes taken from the chaotic map.

\section{DISCUSSION}

We have investigated the dynamics of simple neuronal architecture comprising two interacting neurons. One of the neurons is oscillating sending to the other one a periodic spike train. The signal is transmitted through unidirectional coupling. Such an interaction can be a simplest view on a synaptic transmission when the incoming pulse causes the potential changes (postsynaptic potential) at the receiver cell. If this change exceeds the excitation threshold the cell exhibits a response pulse or spike. We have shown that the response spike sequences can be extremely diverse. Note that in such a master-slave architecture the timing properties of the stimulation (master) signal are fixed and the response is only controlled by the coupling parameter. The units have close parameters, hence operate at close characteristic time scales (pulse duration, inter-spike interval). Then the model gives a simplified view on communication between neurons with similar properties. It appears that internal dynamical properties of the MFHN neuron play a crucial role in the response dynamics. The presence of excitation threshold gives the possibility of long lasting subthreshold oscil- 


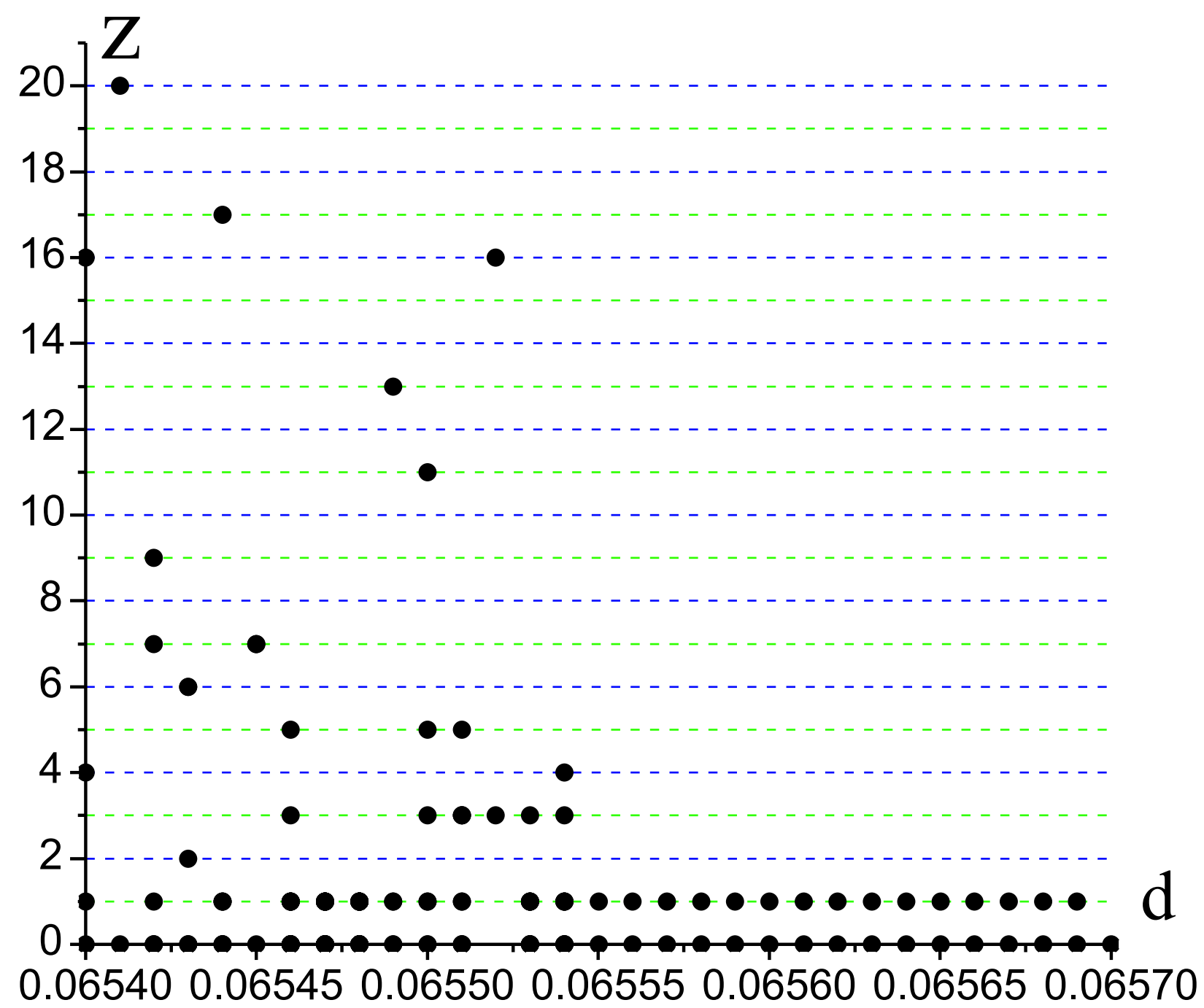

FIG. 11: Bifurcation diagram (FIG. 5) for digital variable $z$.

lations. Moreover, when stimulated periodically the stable and unstable manifolds of the saddle point (FIG. 1 (b)), that define the excitation threshold, may form a complex homoclinic structures in the three-dimensional non-autonomous phase space. The presence of such structures can be noted in FIG. 6 where the response points form two inter-related scrolls with one corresponding to the subthreshold signal and the other to the spiking mode.

We have shown that the spiking response can be approximately described by 1D phase map. The PRC function has been obtained. PRC analysis has shown major bifurcation scenarios of chaotic response. The map displays different kinds of intermittency, complex periodic orbits and chaotic attractors. We have also described how the spiking response can be associated with digital sequences. Such sequences can be associated with a kind 


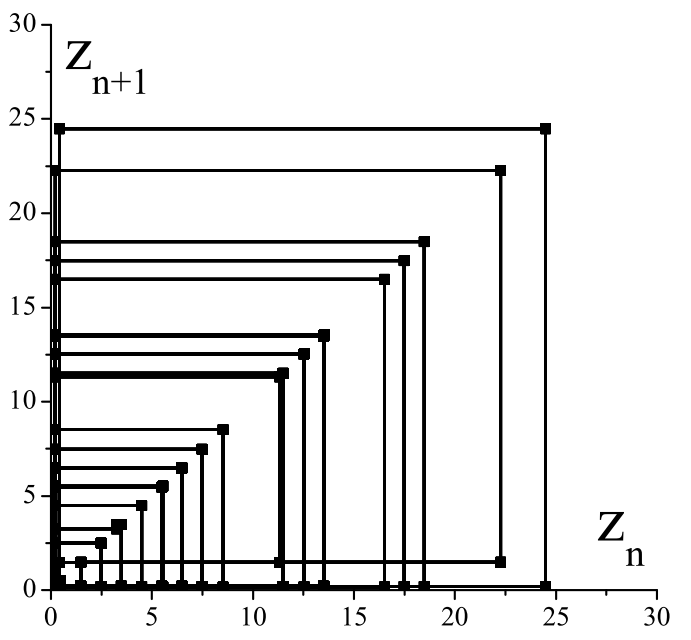

(a)

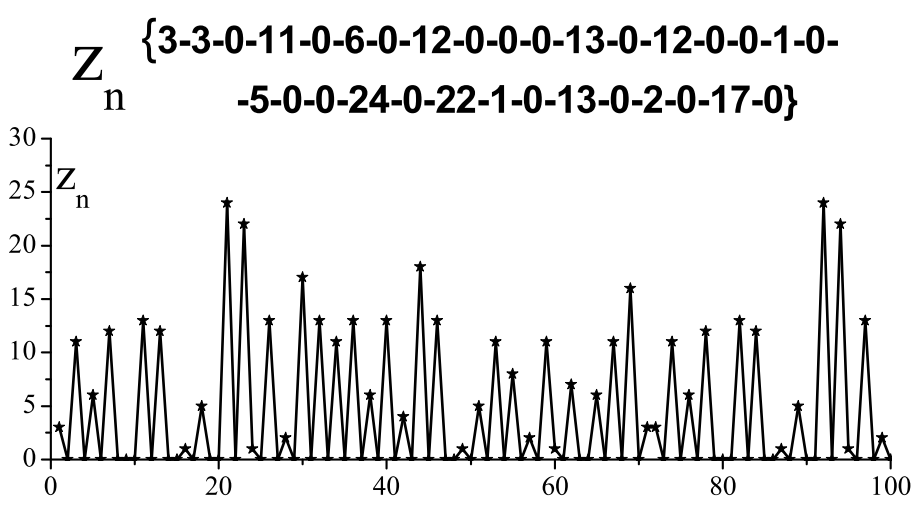

(b)

FIG. 12: (a) A chaotic route for digital variable $z$ in $\left(z_{n}, z_{n+1}\right)$-plane. (b) Digital sequence $\left\{z_{n}\right\}$. Parameter values: $\epsilon=0.441, I_{m}=0.218, I_{s}=0.21, d=0.06543$.

of "spike-communication-code" that could be used for internal representation of sensorial information in the neuronal systems. The information can be transformed in a "spiking route" that is controlled by the interaction strength and can be found by appropriate reset of initial spiking phase.

Finally, we have presented analog electronic circuit reproducing the master-slave neuronal configuration. The results obtained have confirmed the theoretical predictions. Spiking phase maps of regular and chaotic structure have been obtained experimentally. Indeed, the phenomena of coupling-controlled spiking response has robust properties and can be useful in perspective design of neurodynamic-based information processing systems.

\section{Acknowledgments}

This research was supported in part by Russian Foundation for Basic Research (grants 03-02-17135), by grant of President of Russian Federation (MK 4586.2004.2). V.B.K. acknowledges Russian Science Support Foundation for financial support.

[1] E.R. Kandel, J.H. Schwartz, T.M. Jessell (Eds.) Principles of Neural Science. Third Edition (Prentice-Hall Intern. Inc., 1991). 
[2] A. T. Winfree, The Geometry of Biological Time (Springer- Verlag, New York, 1980).

[3] R. Llinas, I of the Vortex: From neurons to self (The MIT Press, Massachusets, 2001).

[4] I. Sugihara, E.J. Lang and R. Llinas, J. Physiology 470, 243 (1993).

[5] E. Leznik, V. Makarenko, and R. Llinas, J. Neurosci. 22, 2804 (2002).

[6] Z. Emri et al., J. Neurosci., 98, 111 (2000); K. Antal, Z. Emri, and V. Crunelli, Thalamus \& Related Systems 1, 105 (2001).

[7] K. J. Suter and D. Jaeger, J. Neurosci., 124, 305 (2004).

[8] D.A. Henze, G. Buzsáki, International Congress Series 1250, 161 (2003).

[9] J.C. Magee, Trends Neurosci. 26 (1), 14 (2003); O. Melamed et al. Trends Neurosci. 27 (1), 11 (2004).

[10] N. Brenner et al., Neural Computation 12, 1531 (2000).

[11] C. Panchev and S. Wermter, Neurocomputing 5860, 365 (2004)

[12] E.M. Izhikevich, N.S. Desai, E.C. Walcott and F. C. Hoppensteadt, Trends Neurosci. 26 (3) (2003); E.M. Izhikevich, Neural Networks 14, 883 (2001).

[13] V.B. Kazantsev, Phys. Rev. E 64, 056210 (2001).

[14] L. Glass, M.C. Mackey, From Clocks to Chaos: The Rhythms of Life (Princeton University Press, 1988); L. Glass, Nature 410, 277 (2001).

[15] L. Glass, and M.E. Josephson, Phys. Rev. Lett. 75 (10), 2059 (1995); L. Glass et al., Phys Rev E 65021908 (2002).

[16] K. Yoshino, T. Nomura, K. Pakdaman, and S. Sato, Phys. Rev. E 59, 956 (1999); K. Pakdaman, Phys Rev E 63, 041907 (2001).

[17] R. FitzHugh, Biophys. J. 1, 445 (1961).

[18] E.M. Izhikevich, Int. J. Bifurc. Chaos 10(6), 1171 (2000);

[19] J. Rinzel and B.B. Ermentrout, in Methods in neuronal modelling, edited by C. Koch and I. Segev (MIT press, Cambridge Massachussetts, 1998), pp. 251-292.

[20] S. Binczak, V.B. Kazantsev, V.I. Nekorkin, and J.M. Bilbault, Electron. Lett. 39, 13 (2003).

[] The value $I^{*}(\epsilon)$ corresponds to the appearance of stable limit cycle on the phase plane. Depending on $\epsilon$ it can appear through big separatrix loop bifurcation or saddle-node limit cycle bifurcation. 